\title{
DESCRIPCION DE HUEVOS Y LARVAS DE PRIMER ESTADIO DEL GENERO GYRIOSOMUS GUERIN-MENEVILLE, 1834 (COLEOPTERA, TENEBRIONIDAE, NYCTELIINI)
}

\author{
DESCRIPTION OF EGGS AND FIRST INSTAR LARVAE OF THE GENUS \\ GYRIOSOMUS GUERIN-MENEVILLE, 1834 \\ (COLEOPTERA, TENEBRIONIDAE, NYCTELIINI)
}

\author{
Jaime Pizarro-Araya $^{1,2}$, Viviane Jerez ${ }^{1} \&$ Jorge Cepeda-Pizarro ${ }^{2}$ \\ ${ }^{1}$ Departamento de Zoología, Facultad de Ciencias Naturales y Oceanográficas, Universidad de Concepción, Casilla \\ 160-C, Concepción, Chile.Email: jaimepizarro@udec.cl; vijerez@udec.cl \\ ${ }^{2}$ Laboratorio de Entomología Ecológica, Departamento de Biología, Facultad de Ciencias, Universidad de La Serena, \\ Casilla 599, La Serena, Chile. Email: jcepeda@userena.cl
}

\begin{abstract}
RESUMEN
Se describe la estructura y ornamentación del exocorion del huevo de siete especies del género Gyriosomus GuérinMéneville, 1834: G. impressus, G. luczotii, G. elongatus, G. whitei, G. subrugatus, G. curtisi y G. batesi, la morfología del primer estadio larvario de G. luczotii y G. subrugatus y se entregan nuevos antecedentes para G. kingi. Para la obtención de huevos y larvas de estas especies se recolectó en terreno parejas que fueron mantenidas en cajas de crianza hasta la ovipostura y posterior eclosión. La estructura y ornamentación del exocorion del huevo y características morfológicas externas de la larva fueron analizadas mediante microscopía electrónica de barrido. Los resultados muestran que los huevos de Gyriosomus tienen una micropila redondeada y exocorion liso, con células hexagonales sin aeropilas y no se observó diferencias interespecíficas para estos caracteres. La larva de primer estadio presenta características morfológicas adaptativas para la vida edáfica: patas protorácicas de función cavadora, cápsula cefálica con gran cantidad de sensillas y pigopodio bien desarrollado. Se analiza la importancia de algunos caracteres morfológicos de la larva de primer estadio como criterio de diagnóstico específico y se establece que las diferencias interespecíficas referidas a las sensillas frontales, forma del clípeo y margen anterior del labro tienen valor taxonómico y probablemente fílogenético.
\end{abstract}

Palabras Claves: Tenebrionidae, Nycteliini, Gyriosomus, exocorion, larva de primer estadio.

\section{ABSTRACT}

The egg structure and ornamentation of seven species of Gyriosomus Guérin-Méneville, 1834 are described and analyzed: G. impressus, G. luczotii, G. elongatus, G. whitei, G. subrugatus, G. curtisi y G. batesi and the morphology of the first instar larvae of G. luczotii y G. subrugatus. To the obtain eggs and the first instar larvae, couples of G. impressus, $G$. luczotii, G. elongatus, G. whitei, G. subrugatus, G. curtisi and G. batesi, were collected in the field and maintained in rearing cages until the emergence of larvi. Both the egg structure and the ornamentation and the morphological characteristics of the larvae were studied by scanning electronic microscopy. While the egg description involved the 8 species, larval morphology was studied in G. luczotii and G. subrugatus. Results show that Gyriosomus eggs have a rounded micropyle and a smooth exochorion, composed by hexagonal cells without aeropyles. No interspecific differences were observed with respect to these characteristics. Gyriosomus larvae shows morphological characteristics suited for an edaphic life, such as the presence of strong digging legs, particularly the prothoracic, abundant sensillar provision, and a well developed pigopodium. Interspecific differences referred to jaw, anterior part of labrum, and tarsungulus have taxonomic value and a possible phylogenetic meaning. The importance of larval morphology as an element for 2 specific diagnosis is analyzed.

KeYwords: Tenebrionidae, Nycteliini, Gyriosomus, exochorion, first instar larvae. 


\section{INTRODUCCION}

Los coleópteros de la familia Tenebrionidae presentan estrategias adaptativas especializadas a ecosistemas terrestres con condiciones de sequedad, mediante el desarrollo de estructuras morfológicas particulares (Keleynikova 1963; Iwan \& Becváø 2000; Duncan 2003; Byrne \& Duncan 2003; Herrera-Vega \& Sánchez-Piñero 2004) y/o a la presencia de estrategias etológicas evasivas a las altas temperaturas (Flores 1998), por lo cual constituyen un componente significativo de la biota de los ecosistemas áridos y semiáridos (Cloudsley-Thompson 1975; Cepeda-Pizarro 1989; Crawford et al. 1993). Por otra parte, tanto larvas como adultos participan en la descomposición de materia orgánica, y constituyen una fuente importante de proteínas para la alimentación de vertebrados e invertebrados propios de estos ecosistemas (Keleynikova 1970; Wise 1981; Crawford et al. 1993).

El conocimiento de los estados preimaginales de Tenebrionidae reviste un notable interés referido a estudios sobre biología y ecología del suelo, debido al desarrollo hipogeo de sus estadios larvarios (Flores 1998). Por otra parte, en diversos grupos de insectos los caracteres morfológicos de los estadios larvarios permiten reconocer diferencias poblacionales (Horsfall et al. 1970; Francisco \& do Prado 2001), postular inferencias filogenéticas al ser utilizados como homologías (Stark \& Szczytko 1982; Aalbu 1985; Schunger et al. 2003; Bouchard \& Steiner 2004; Beutel \& Friedrich 2005) o bien evidenciar adaptaciones ontogénicas a hábitats particulares (Luff 1981; Pizarro-Araya et al. 2003).

Sin embargo en relación con los tenebriónidos chilenos, existen escasos antecedentes acerca de su biología (Mondaca 2004; Pizarro-Araya et al. 2005) y la mayoría de los trabajos han considerado aspectos morfológicos sólo de pupas y larvas de tercer estadio de unas pocas especies (Artigas \& Brañas-Rivas 1973; Cekalovic \& Quezada 1973, 1982; Cekalovic \& Morales 1974).

Entre los coleópteros propios de ecosistemas desérticos costeros de Chile se destaca el género Gyriosomus Guérin-Méneville, 1834 (Tenebrionidae: Nycteliini), taxa endémico y erémico, con 38 especies distribuidas entre las provincias biogeográficas de los Desiertos Costeros e Interior y Chilena Central (Mondaca 2004; Pizarro-Araya \& Flores 2004; Pizarro-Araya \& Jerez 2004, Pizarro-Araya et al. 2004, 2005).
Los objetivos del presente trabajo son por lo tanto describir y comparar la estructura del exocorion del huevo de G. impressus Guérin-Méneville, 1834, G. luczotii Laporte, 1840, G. elongatus Waterhouse, 1843, G. whitei Waterhouse, 1844, G. subrugatus Fairmaire, 1876, G. curtisi Fairmaire, 1876, G. batesi Fairmaire, 1876, describir la morfología del primer estadio larvario de G. kingi, G. luczotii y $G$. subrugatus y entregar nuevos caracteres para G. kingi (Pizarro-Araya et al. 2003) con el fin de utilizarlos como elementos de diagnóstico específico para las especies adscritas al género Gyriosomus.

\section{MATERIALES Y METODOS}

El material estudiado proviene de parejas de adultos recolectadas en las siguientes localidades y fechas: $G$. curtisi: Paposo $25^{\circ} 00^{\prime} \mathrm{S}, 70^{\circ} 28^{\prime} \mathrm{W}, 30 \mathrm{msnm}$, 10.XII.2002; G. batesi: Algarrobal $28^{\circ} 07^{\prime} \mathrm{S}, 70^{\circ} 46^{\prime}$ W, 350 msnm, 03.XI.2002; G. subrugatus: Chacritas, $28^{\circ} 23^{\prime} \mathrm{S}, 70^{\circ} 42^{\prime} \mathrm{W}, 644 \mathrm{msnm}, 03 . X \mathrm{X} .2002$; G. whitei: Chañaral del Aceituno, $29^{\circ} 02^{\prime} \mathrm{S}, 71^{\circ} 25^{\prime} \mathrm{W}, 91 \mathrm{msnm}$, 06.XI.2002; G. elongatus: Isla Gaviota, $29^{\circ} 15^{\prime} 14,9^{\prime}$ S, $71^{\circ} 28^{\prime} 15,9^{\prime} \mathrm{W}, 80 \mathrm{msnm}, 07 . X I .2002 ; G$. impressus: Los Choros, $29^{\circ} 21^{\prime} \mathrm{S}, 71^{\circ} 10^{\prime} \mathrm{W}, 300$ msnm, 07.XI.2002 y G. luczotii: Colina El Pino, La Serena, $29^{\circ} 54^{\prime} \mathrm{S}, 71^{\circ} 15^{\prime} \mathrm{W}, 90 \mathrm{msnm}, 20 . \mathrm{XI} .2003$. Las parejas se mantuvieron en cajas de crianza con sustrato de arena, temperatura de $17^{\circ} \mathrm{C}$ mínima y $24^{\circ}$ C máxima y fotoperíodo de 12 horas luz y 12 horas oscuridad, hasta la oviposición y posterior eclosión. Los insectos fueron alimentados con Erodium cicutarium (L.) (Geraniaceae) y Cristaria glaucophylla Cav. (Malvaceae) más un suplemento de dieta artificial.

Los huevos y larvas de primer estadio obtenidos fueron fijados en alcohol $70 \%$ y posteriormente deshidratados en batería de alcohol, secado punto crítico y metalizado con oro, según la técnica descrita por Jerez (2003) y Pizarro-Araya et al. (2003), para su observación y obtención de fotografías a microscopio electrónico de barrido (ETEC Autoscann $\mathrm{U}_{1}$ ) del Laboratorio de Microscopía Electrónica de la Universidad de Concepción. Para la descripción de la estructura y ornamentación del exocorion se utilizó la nomenclatura seguida por Jerez (2003) y Pizarro-Araya et al. (2003). Para la morfología larvaria se siguió a Keleynikova (1963, 1970), López-Sánchez et al. (1985a, 1985b, 1987), Doyen $(1988,1993)$ y Pizarro-Araya et al. (2003). 
Descripción de huevos y larvas del género Gyriosomus: PizArRo-AraYA, J. ET AL.

\section{RESULTADOS}

DESCRIPCIÓN DEL HUEVO

Cada ovipostura consta de 7 a 10 huevos unidos lateralmente y cada huevo está recubierto por una película de arena adherida al corion mediante un mucílago producido por las hembras al momento de la oviposición. El huevo presenta forma elongada, polos redondeados, exocorion de color blanco, liso y sin aeropilas (Fig. 1). El primer tercio del huevo está ornamentado con celdas subhexagonales con un diámetro de $18.2 \mu$ (rango: 11.8 - $24.6 \mu ; \mathrm{n}=5$ ) y delimitadas por bordes solevantados (Fig. 2). Distribuidos homogéneamente en el exocorion se encuentran cuerpos elevados de distinto tamaño y de aspecto redondeado, ubicados al interior y en los bordes de las celdas (Fig. 3). El área micropilar de forma circular y ligeramente sobresaliente se encuentra en la región apical del huevo, con un diámetro de $18.7 \mu$ (rango: 12.3 - $22.9 \mu ; \mathrm{n}=5$ ) y presenta numerosos poros de diámetro $1.55 \mu$ (rango: $0.8-1.5 \mu ; \mathrm{n}=5)$. La micropila presenta además aeropilas con diámetro aproximado de $1.5 \mu$ (rango: 1 - $2 \mu ; \mathrm{n}=5$ ) (Fig. 4).

\section{LARVA DE PRIMER ESTADIO}

Diagnosis: La larva de G. luczotii y G. subrugatus presenta el cuerpo elongado y cilíndrico con una longitud de $4.8 \mathrm{~mm}$ (rango: 2.8 - $6.8 \mathrm{~mm} ; \mathrm{n}=45$ ) (Fig. 5). Región frontal deprimida dorsalmente y provista de numerosas sensillas dispuestas en hileras horizontales (Fig. 6). Patas protorácicas duplican en tamaño a las meso y metatorácicas (Fig. 15).

Descripción. CABEZA: globosa y fuertemente esclerotizada con sutura epicraneal no evidente; epicranio presenta setas dorsales y laterales largas y densas (Fig. 6); sutura frontal rectilínea; sutura frontoclipeal notoria y ligeramente curvada. Región frontal deprimida dorsalmente y provista de numerosos tubérculos de ápice redondeado y dispuestos en hileras horizontales (Figs. 7, 8). Ocelos ausentes. Antena con tres segmentos, primer segmento antenal alargado, segundo igual o más largo que la mitad del primero; tercer segmento pequeño y lleva una sensilla redondeada y una seta apical (Fig. 10). Clípeo subrectangular y glabro (Fig. 7). Labro transverso, con un par de sensillas dorsales y un pecten formado por una hilera de setas gruesas; margen distal levemente sinuado y provisto de setas largas y rígidas (Figs. 8, 13). Mandíbulas subtriangulares, quitinizadas y con dos dientes apicales de ápice obtuso (Fig. 11); mola bien desarrollada, ovalada, cóncava y con superficie lisa; porción basolateral de la superficie dorsal con un mechón de setas largas y rígidas. Palpos maxilares trisegmentados, último segmento corto y de ápice redondeado (Fig. 12); mala redondeada y provista de numerosas setas cortas y gruesas en el margen externo. Palpos labiales con dos segmentos (Fig. 12). Tórax. Primer segmento más largo que los siguientes; escudo protorácico subrectangular y curvado lateralmente. Meso y metatórax posee tegumento poco esclerotizado, y ruptor ovi no visible. Patas protorácicas más desarrolladas que el segundo y el tercer par (Fig. 15); coxa de forma tetragonal, un poco más ancha que larga; margen interno de la tibia lleva 3 espinas de aspecto lanceolado; margen anterior y superficie externa con numerosas espinas largas y delgadas; margen interno del fémur lleva una hilera de espinas cortas, gruesas y de ápice obtuso; tarsungulus largo y curvado (Fig. 16). Espiráculos mesotorácicos de diámetro circular, uniforos y peritrema oscuro (Fig. 17). Abdomen. Segmentos con pilosidad escasa y corta; IX segmento abdominal alargado y subtriangular, con el extremo apical redondeado; dorsalmente el tergito está provisto de cerdas largas repartidas por toda la superficie; pigopodio bien desarrollado y fuertemente esclerotizado en sus extremos (Fig. 18).

\section{DIFERENCIAS INTERESPECÍFICAS}

Las larvas de primer estadio de las dos especies de Gyriosomus descritas en este estudio (G. luczotii y $G$. subrugatus) muestran uniformidad en los caracteres morfológicos con respecto a la larva de G. kingi descrita por Pizarro-Araya et al. (2003). Sin embargo se observan algunas diferencias en la región frontal y aparato bucal. La frente de G. kingi y G. luczotii está provista de numerosos tubérculos dispuestos en hileras horizontales (Figs. 7,8) que en G. subrugatus se observan pequeños y dispersos (Fig. 9). El clípeo puede ser subrectangular como en $G$. kingi (Fig. 7), subrectangular pero con el margen distal cóncavo como en G luczotii (Fig. 8), o subrectilíneo y con algunos tubérculos dorsales como en $G$ subrugatus (Fig. 9). El labro es levemente sinuado en G. kingi (Fig. 7) y emarginado en G luczotii (Fig. 13) y G subrugatus (Fig. 9). El último segmento de los palpos maxilares puede ser corto y de ápice redondeado como en G. kingi (Fig. 7), márgenes del segundo segmento con seta apical, primer segmento provisto de un sensorio redondeado y setas apicales como en G. luczotii (Fig. 11), o elongado y de ápice redondeado como en G. subrugatus (Fig. 12). 

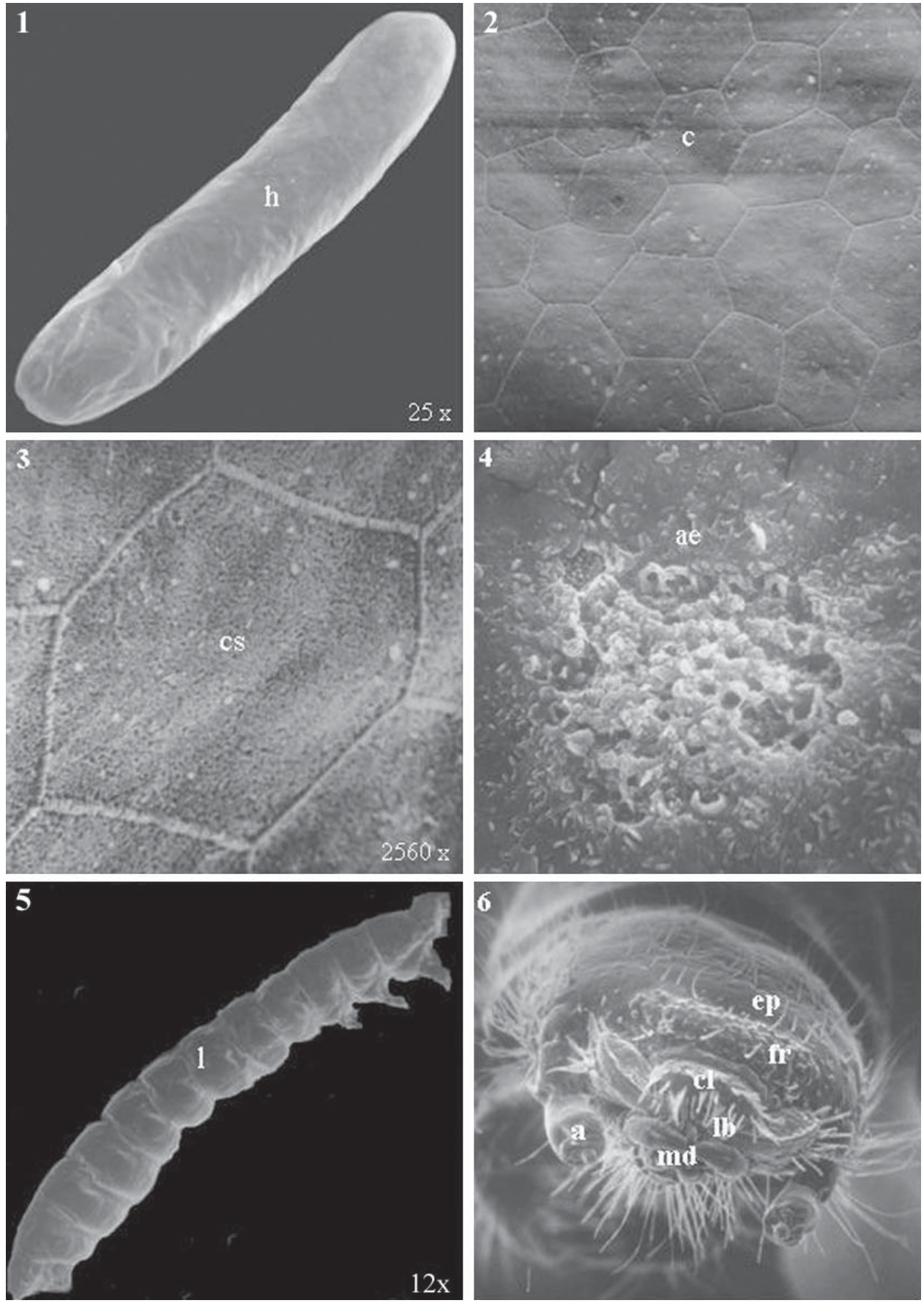

Figuras 1-6. 1. G. kingi, huevo vista lateral. 2. G. subrugatus, detalle del corion. 3. G. kingi, cs: celda subhexagonal. 4. G. batesi, área micropilar. ae: aeropilas. 5. G. kingi, larva de primer estadio, vista lateral. 6. G. subrugatus, cápsula cefálica, a: antena, cl: clípeo, ep: epicranio, fr: frente, lb: labro, md: mandíbula.

Figures 1-6. 1. G. kingi, egg lateral view. 2. G. subrugatus, detail of chorion. 3. G. kingi, cs: subhexagonal cell. 4. G. batesi, micropylar area. ae: aeropyles. 5. G. kingi, first instar larvae, lateral view. 6. G. subrugatus, cephalic capsule. a: antenna, cl: clipeus, ep: epicranium, fr: frons, lb: labrum, md: mandibles. 

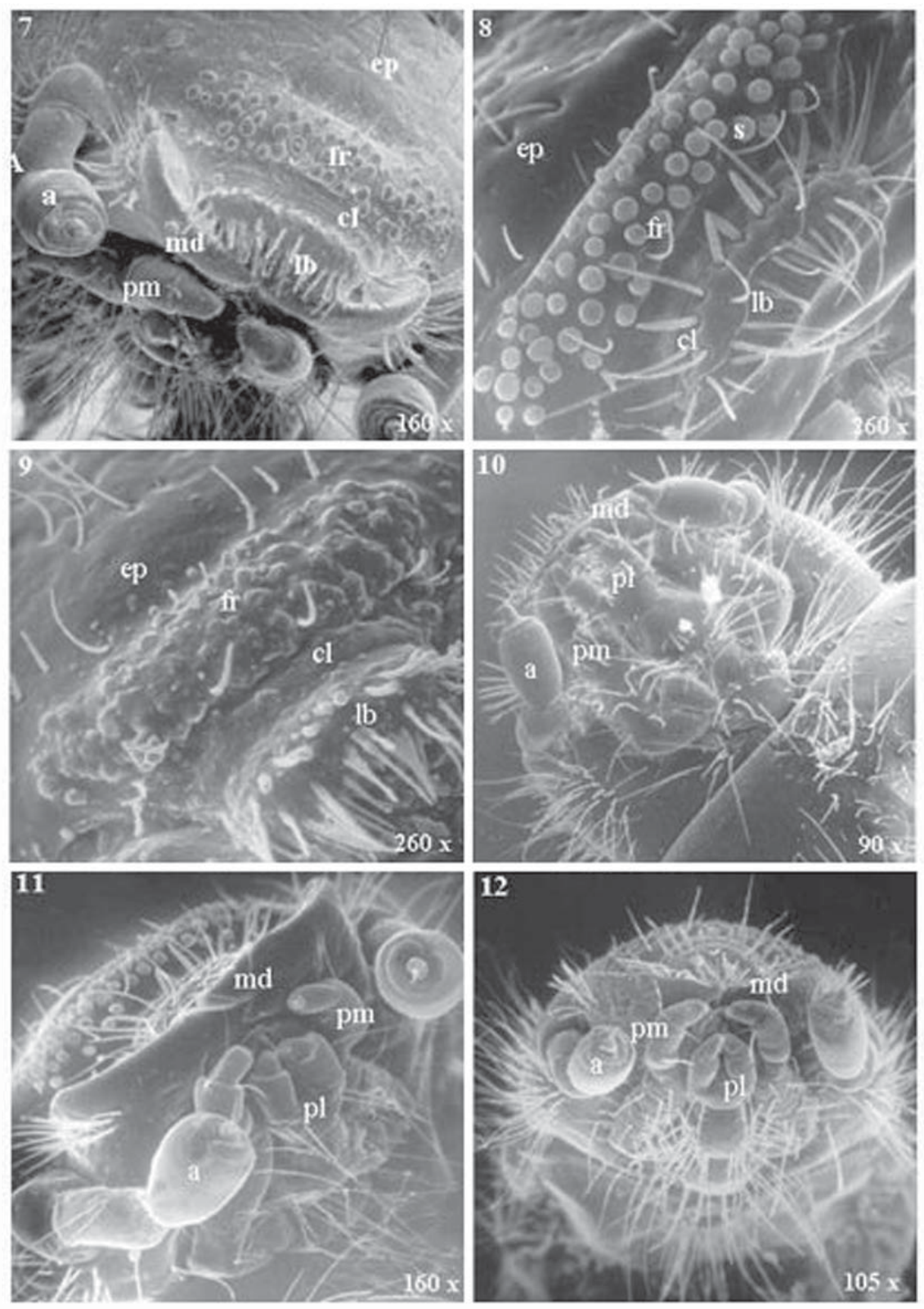

Figuras 7-12. Larva de primer estadio, detalles cápsula cefálica. 7. G. kingi, a: antena, cl: clipeo, ep: epicranio, fr: frente, lb: labro, md: mandíbulas, pm: palpo maxilar. 8. G. luczotii, ep: epicranio, cl: clípeo, fr: frente, lb: labro, s: sensilla 9. G. subrugatus, cl: clípeo, ep: epicranio, fr: frente, lb: labro. 10. G. kingi, complejo máxilo-labial, vista ventral, a: antena md: mandíbula, pm: palpo maxilar, pl: palpo labial. 11. G. luczotii, complejo máxilo-labial vista ventral, a: antena, md: mandíbula, pm: palpo maxilar, pl: palpo labial. 12. G. subrugatus, complejo maxilo-labial, vista ventral, a: antena, md: mandíbula, pm: palpo maxilar, pl: palpo labial.

FigURES 7-12. First instar larvae, details of cephalic capsule. 7. G. kingi, a: antenna, cl: clipeus, ep: epicranium, fr: frons, lb: labrum, md: mandibles, pm: maxillary palpi. 8. G. luczotii, ep: epicranium, cl: clipeus, fr: frons, lb: labrum, s: sensilla. 9. G. subrugatus, cl: clipeus, ep: epicranium, fr: frons, lb: labrum. 10. G. kingi, maxillo labila complex, a: antenna md: mandible, pm: maxillary palpi, pl: labial palpi. 11. G. luczotii, maxillo-labial complex, a: antenna, md: mandible, pm: maxillary palpi, pl: labial. 12. G. subrugatus, complex maxillo-labial, a: antenna, md: mandible, pm: maxillary palpi, pl: labial palpi. 

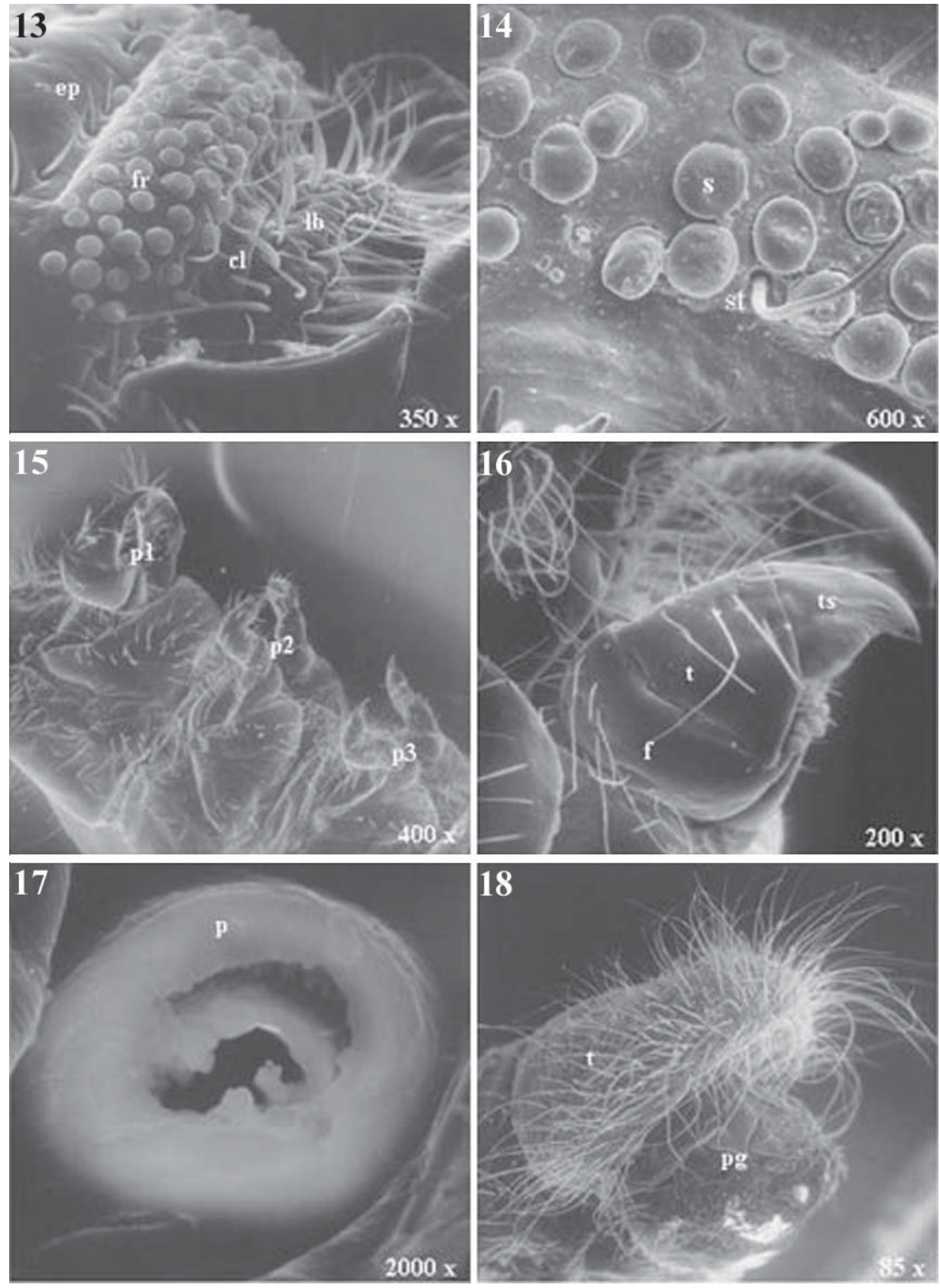

FiguRAs 13-18. 13. G. luczotii, capsula cefálica, vista latero frontal. ep: epicranio, fr: frente, cl: clípeo, lb: labro. 14. G. kingi, detalle frente, s: sensillas, st: seta frontal. 15. G. kingi, larva de primer estadio vista lateral, p1: pata protorácica, p2: pata mesotorácica, p3: pata metatorácica. 16. G. luczotii, pata protorácica, f: fémur, t: tibia, ts: tarsungulus. $17, G$. kingi, espiráculo mesotorácico, p: peritrema. 18. G. subrugatus, placa tergal del VIII y IX segmentos abdominales, t: tergito (VIII), pg: pigopodio (IX)

FigURES 13-18. 13. G. luczotii, cephalic capsule, lateral and frontal view. ep: epicranium, fr: frons, cl: clipeus, lb: labrum. 14. G. kingi, frons detail, s: sensilla, st: frontal setae. 15. G. kingi, first instar larvae, p1: prothoracic leg, p2: mesothoracic leg, p3: metathoracic leg. 16. G. luczotii, prothoracic leg detail, f: femur, t: tibiae, ts: tarsungulus. 17, G. kingi, mesothoracic spiracle, p: peritreme. 18. G. subrugatus, VIII tergal plate and abdominal segments IX, t: tergite (VIII), pg: pygopods (IX). 


\section{DISCUSION}

En insectos y especialmente en Coleoptera, el conocimiento de la estructura coriónica y de la morfología larvaria permite establecer caracteres de diagnóstico a nivel de familia, género y especie (Crowson 1981). En el caso particular de Gyriosomus, sólo se conocían aspectos morfológicos y bionómicos para G. kingi descritos por Pizarro-Araya et al. (2003), desconociéndose aspectos taxonómicos, ontogénicos y biológicos de las restantes especies. Los resultados obtenidos en este estudio pueden contribuir a definir la posición sistemática de este taxón y sus reales vinculaciones con los demás elementos de Nycteliini (Pizarro-Araya et al. 2003).

En relación con la estructura coriónica de los huevos, no se encontraron diferencias interespecíficas entre las siete especies analizadas (G. impressus, G. luczotii, G. elongatus, G. whitei, G. subrugatus, G. curtisi y G. batesi) y la de G. kingi descrita anteriormente por Pizarro-Araya et al. (2003). Por otra parte, la ausencia de descripciones de huevos de otros Nycteliini y/o tribus hermanas (e.g. Praocini y Physogasterini) no permite establecer inferencias filogenéticas basadas en estos caracteres.

En relación con el estado larvario, la larva de primer estadio de Gyriosomus corresponde al tipo pedobionta propuesta por Keleynikova (1963) y se adscribe a la descripción morfológica señalada por Doyen $(1988,1993)$ para larvas de la subfamilia Pimeliinae, en el sentido de presentar estructuras adaptativas para la vida edáfica, tales como son la abundante dotación sensilar en la cápsula cefálica, patas cavadoras (especialmente las protorácicas) y pigopodio bien desarrollado.

Para G. luczotii, G. subrugatus y G. kingi, la forma de las sensillas frontales y características del clípeo y labro de la larva de primer estadio destacan como caracteres de valor taxonómico y posiblemente filogenético. Finalmente los resultados obtenidos en este estudio podrán ser complementados con futuras investigaciones acerca de los estados preimaginales de los demás elementos de Gyriosomus, antecedentes que permitirán comprobar o rechazar las hipótesis de monofilia que existen para Gyriosomus y los demás géneros de Nycteliini.

\section{AGRADECIMIENTOS}

Los autores agradecen al personal del Laboratorio de Microscopía Electrónica de la Dirección de Investigación de la Universidad de Concepción, Concepción, Chile. Este trabajo fue financiado por los proyectos DIUC N ${ }^{\circ}$ 203.113.061-1.0, Dirección de Investigación, Universidad de Concepción, Concepción, Chile y DIULS N ${ }^{\circ} 220.2 .17$, Dirección de Investigación, Universidad de La Serena, La Serena. Chile.

\section{BIBLIOGRAFIA}

AALBU, R.L. 1985. New genus and species of Triorophini, including immatures, reproductive structures, and notes on biology and phylogeny (Coleoptera: Tenebrionidae). Ann. Entomol. Soc. Am. 78: 541553.

Artigas, J. \& P. Brañas-Rivas. 1973. Descripción de la larva y pupa de Praocis curta Solier (Coleoptera: Tenebrionidae). Bol. Soc. Biol. Concepción. XLVI: 64-74.

Beutel, R.G. \& F. Friedrich. 2005. Comparative study of larvae of Tenebrionoidea (Coleoptera: Cucujiformia). Eur. J. Entomol. 102: 241-264.

Bouchard, P. \& W.E. Steiner. 2004. First descriptions of Coelometopini pupae (Coleoptera: Tenebrionidae) from Australia, Southeast Asia and the Pacific region, with comments on phylogenetic relationships and antipredator adaptations. Syst. Ent. 29: 101-114.

Byrne, M.J. \& F.D. Duncan. 2003. The role of the subelytral spiracles in respiration in the flightless dung beetle Circellium bacchus. J. Exp. Biol. 206: 1309-1318.

Cekalovic, T. \& A. Quezada. 1973. Descripción de la larva, pupa y distribución geográfica de Emmallodera multipunctata curvidens Kulzer, 1955 (Coleoptera: Tenebrionidae). Anal. Inst. Patagonia. 4: 417-422.

Cekalovic, T. \& A. Quezada. 1982. Descripción de la larva de Nycterinus (Eunycterinus) abdominalis Eschscholtz, 1828 (Coleoptera, Tenebrionidae). Bol. Soc. Biol. Concepción. LIII: 47-51.

Cekalovic, T. \& E. Morales. 1974. Descripción de la larva y pupa de Oligocara nitida Solier, 1848 (Coleoptera: Tenebrionidae). Bol. Soc. Biol. Concepción. XLVIII: 173-177.

Cepeda-Pizarro, J.G. 1989. Actividad temporal de tenebriónidos epígeos (Coleoptera) y su relación con la vegetación arbustiva en un ecosistema árido de Chile. Rev. Chil. Hist. Nat. 62: 115-125.

Cloudsley-Thompson, J.L. 1975. Adaptations of Arthropoda to arid environments. Annu. Rev. Entomol. 20: 261-283.

Crawford, C.S., W.P. Mackay \& J. Cepeda-Pizarro. 1993. 
Detritivores of the Chilean arid zone $\left(27-32^{\circ} \mathrm{S}\right)$ and the Namib desert: a preliminary comparison. Rev. Chil. Hist. Nat. 66: 283-289.

Crowson, R.A. 1981. The biology of the Coleoptera. Academic Press, London. 802 pp.

DOYEN, J.T. 1988. Descriptions of some phylogenetically important larvae of Tenebrionidae (Coleoptera). Coleopt. Bull. 42: 285-301.

DoYen, J.T. 1993. Cladistic relationships among Pimeliinae Tenebrionidae (Coleoptera). J. New York Ent. Soc. 101: 443-514.

Duncan, F.D. 2003. The role of the subelytral cavity in respiration in a tenebrionid beetle, Onymacris multistriata (Tenebrionidae: Adesmiini). J. Insect Physiol. 49: 339-346.

Flores, G.E. 1998. Tenebrionidae. En: Morrone JJ \& S Coscarón (eds.). Biodiversidad de artrópodos argentinos: una perspectiva biotaxonómica: 232257. Ediciones Sur. Buenos Aires, Argentina. vii $+599 \mathrm{pp}$.

Francisco, O. \& A.P. do Prado. 2001. Characterization of the larval stages of Alphitobius diaperinus (Panzer) (Coleoptera: Tenebrionidae) using head capsule width. Rev. Bras. Biol. 1: 125-131.

Herrera-Vega, J.M. \& F. Sánchez-Piñero. 2004. Description of the last larval stage of Heliotaurus ruficollis Fabricius, 1781 (Coleoptera: Tenebrionidae: Alleculinae). Ann. Zool. 54: 729734.

Horsfall, W.R., F.R. Voorhess \& E.W. Cupp. 1970. Eggs of floodwater mosquitoes. XIII. Chorionic sculpturing. Ann. Entomol. Soc. Amer. 63: 17091716.

IWAN, D. \& S. BECVÁø. 2000. Description of the early stages of Anomalipus plebejus plebejulus (Coleoptera: Tenebrionidae) from Zimbabwe with notes on the classification of the Opatrinae. Eur. J. Entomol. 97: 403-412.

JEREZ, V. 2003. Interspecific differentiation in eggs and first instar larvae in the genus Procalus Clarck 1865 (Chrysomelidae: Alticinae), p. 147-153. En: Furth D.G. (ed.). Special Topics in Leaf Beetles Biology, Proc. $5^{\text {th }}$ Sym. on the Chrysomelidae. XXI International congress of Entomology. Foz do Iguassu, Brazil 2000.

KelEyNIKOVA, S.I. 1963. Larval types of palearctic darkling beetles (Coleoptera, Tenebrionidae). Ent. Rev. 42: 292-296.

Keleynikova, S.I. 1970. Darkling beetle larvae of paleartic tribes of the subfamily Tentyriinae (Coleoptera, Tenebrionidae). Ent. Rev. 49: 245-253.

López-SÁnchez, S., A. De los SAntos \& C. Montes. 1985a. Estudio morfológico de los estados preimaginales de Micrositus ulyssiponensis Germ. 1824
(Coleoptera: Tenebrionidae). An. Biol. Univ. Murcia. 3: 95-102.

López-SÁnchez, S., A. de los Santos \& C. Montes. 1985b. Estudio morfológico de la forma larvaria de Tentyria placyteps Stev. (1829) Eos. LXI: 173182.

López-SÁnchez, S., A. de los SAntos \& C. Montes. 1987. Estudio morfológico de los estados preimaginales de Pimelia costata Waltl. 1835 (Coleoptera: Tenebrionidae). Nouv. Revue Ent. 4: 299-311.

LuFF, M.L. 1981. Diagnostic characters of the eggs of some Carabidae (Coleoptera). Entomol. Scand. Suppl. 15: 317-327.

MondacA, J. 2004. Nueva especie de Gyriosomus GuérinMéneville, 1834 (Coleoptera: Tenebrionidae: Nycteliini) del extremo norte de la Región de Atacama (Chile). Rev. Chil. Ent. 30: 21-26.

Pizarro-Araya, J. \& G.E. Flores. 2004. Two new species of Gyriosomus Guérin-Méneville from Chilean coastal desert (Coleoptera: Tenebrionidae: Nycteliini). J. New York Ent. Soc. 112: 121-126.

Pizarro-Araya, J. \& V. Jerez. 2004. Distribución geográfica del género Gyriosomus Guérin-Méneville, 1834 (Coleoptera: Tenebrionidae): una aproximación biogeográfica. Rev. Chil. Hist. Nat. 77: 491-500.

Pizarro-Araya, J., V. Jerez \& J. Cepeda-Pizarro. 2003. Aspectos bionómicos y descripción de huevos y larvas de primer estadio de Gyriosomus kingi Reed (Coleoptera: Tenebrionidae: Nycteliini). XXV Congreso Nacional de Entomología, Talca, Chile. Libro de Resúmenes: 7.

Pizarro-Araya, J., V. Jerez \& J. Cepeda-Pizarro. 2004. Patrones distribucionales y áreas de endemismo para coleópteros epígeos del desierto costero del norte de Chile (25-32 Lat S). XXVI Congreso Nacional de Entomología, Concepción, Chile. Libro de Resúmenes: 17.

Pizarro-Araya, J., V. Jerez, G.E. Flores \& J. CepedaPizarro. 2005. Taxonomía, biología y distribución geográfica del género endémico chileno Gyriosomus Guérin-Méneville, 1834 (Coleoptera: Tenebrionidae: Nycteliini). VI Congreso Argentino de Entomología, Tucumán, Argentina. Libro de Resúmenes: 111.

Schunger, I., R.G. Beutel \& R. Britz. 2003. Morphology of immature stages of Prostomis mandibularis (Coleoptera: Tenebrionidae: Prostomidae). Eur. J. Entomol. 100: 357-370.

StARK, B.P. \& S.W. SzCZYTKO. 1982. Egg morphology and phylogeny in Pteronarcyidae (Plecoptera). Ann. Entomol. Soc. Amer. 75: 519-529.

WiSE, D.H. 1981. A removal experiment with darkling beetles: Lack of evidence for interespecific competition. Ecology. 62: 727-738. 